\title{
Role of MicroRNAs in Pathophysiology of Non-alcoholic Fatty Liver Disease and Non-alcoholic Steatohepatitis
}

\author{
Farzaneh Iravani ${ }^{1}$, Neda Hosseini ${ }^{2}$, Majid Mojarrad ${ }^{3, *}$
}

1. MSc of human genetics, Department of Medical Genetics, Mashhad University of Medical Sciences, Mashhad, Iran

2. MSc of Molecular and Cellular biology, Medical Genetics Research Center, School of Medicine, Mashhad University of Medical Sciences, Mashhad, Iran

3. Assistant Professor of Medical Genetics, Medical Genetics Research Center, School of Medicine, Mashhad University of Medical Sciences, Mashhad, Iran

\footnotetext{
* Corresponding Author:

Majid Mojarrad, PhD

Department of Medical Genetics, School of Medicine, Mashhad University of Medical Sciences, Mashhad, Iran Telefax: + 985138002243

Email:mojaradm@mums.ac.ir

Received: 27 Feb. 2018

Accepted: 14 Jul. 2018
}

\begin{abstract}
Non-alcoholic fatty liver disease (NAFLD) is the most common liver disorder worldwide. It includes wide range of diseases from different subtypes of simple steatosis to non-alcoholic steatohepatitis (NASH), which may be complicated by liver fibrosis, cirrhosis, or hepatocellular carcinoma. Of the epigenetic factors that play a key role in the progression of it, is microRNAs (miRNAs).

MiRNAs are short non-coding RNAs of 22-23 nucleotides in length, which regulate a large number of genes that have a critical role in regulation of lipid and cholesterol biosynthesis in hepatocytes. MiRNAs can be used as a very powerful biomarker to diagnosis and follow-up any disorder, such as NAFLD and NASH with a high specificity and sensitivity. The aim of this study was to review the role of different miRNAs in the pathophysiology of NASH and NAFLD.
\end{abstract}

KEYWORDS:

MicroRNAs, NAFLD, NASH, Biomarker

Please cite this paper as:

Iravani F, Hosseini N, Mojarrad M. Role of MicroRNAs in Pathophysiology of Nonalcoholic Fatty Liver Disease and Non-alcoholic Steatohepatitis. Middle East J Dig Dis 2018;10:213-219. doi: 10.15171/mejdd.2018.113.

\section{INTRODUCTION}

Non-Alcoholic fatty liver disease (NAFLD) is the most common form of liver diseases in the western countries ${ }^{1-3}$ and its prevalence is increasing dramatically in our country too. ${ }^{4,5}$ The disease occurs as the result of fatty deposition in the liver parenchymal cells (hepatocytes), which cause the wide range of liver abnormalities including steatosis, cirrhosis, and steatohepatitis. ${ }^{6}$ In fact, non-alcoholic steatohepatitis (NASH) is the severe type of NAFLD that can be recognized by intense fibrosis, and steatosis, and usually leads to cirrhosis, and hepatocellular carcinoma (HCC). ${ }^{7}$

The frequency of NAFLD varies from $9 \%$ to $36.9 \%$ according to ethnicity and sex. The prevalence of NAFLD in industrialized countries of North America and European ones have been reported $46 \%$, and $29.9 \%$ of them suffer from NASH. Furthermore, the frequency of NASH is higher among patients with diabetes type II and also obese people, which is high as $76-78 \% .^{8,9}$ Most of the people (48-100\%) suffering from NAFLD show no sign of the disease. So its diagnosis based on liver panel tests happens only when the disease progresses towards advanced stage. ${ }^{6,10}$ Based on the fact that fibrosis and wound arising from hepatocytes damage are not recognizable by photography and normal laboratory tests (aspartate aminotransferase (AST) and alanine aminotransferase (ALT), liver biopsy is the gold standard test in diagnosis of NASH. ${ }^{11}$ 
Molecular studies have shown that NAFLD is a complex disease, which is controlled by both environmental and genetic factors. ${ }^{12}$ In approximately $15-20 \%$ of patients, NAFLD progresses to NASH but mechanisms of this conversion has not been fully understood yet. ${ }^{13}$ The identification of involved molecular mechanisms in the occurrence and development of NAFLD plays a key role in an early diagnosis and even its treatment. Researchers recently have been stated that microRNAs (miRNAs) can contribute to the evolutional etiology of this disease. To support this finding, many experiments have shown vast changes in profile expression of miRNAs in animals suffering from NASH. ${ }^{12,14,15}$

MiRNAs are short non-coding RNAs of 22 nucleotides in length that regulate gene expression extensively. ${ }^{16}$ It has been anticipated that human genome encode about 1000 miRNAs, which are estimated to regulate almost one-third of all human genes. ${ }^{17}$ Thus each mature miRNA could regulate a spectrum of gene targets. ${ }^{18}$ MiRNAs have a post transcriptional impact on gene expression by binding to the 3'- untranslated region of target mRNAs, which either lead to destroying mRNA, or suppressing its translation. ${ }^{19,20}$

Because of well characterized role of miRNAs in organ development, such as liver, it would be obvious that miRNAs can play a central role in different stages of the liver diseases. An increasing number of evidence has supported the role of miRNAs in many human diseases. ${ }^{21-24}$ MiRNAs have been introduced as non-invasive biomarkers of diagnosis due to their secretion into the body fluids and the existence of significant concordance between serum and tissue levels of miRNAs, ${ }^{25,26}$ therefore they could improve diagnosis, prognosis, and management of the disease. ${ }^{27}$

"In the present study, we report the surprising and exciting discovery that serum and plasma contain a large amount of stable miRNAs derived from various tissues/ organs, and that the expression profile of these miRNAs shows great promise as a novel non-invasive biomarker for diagnosis of cancer and other diseases" chen et al, 2008. ${ }^{28}$

After introducing miRNAs as new biomarkers, an increasing number of evidence was published for and against this theory. ${ }^{29-31}$ Based on the fact that miRNAs have been identified in both microvesicles and exosomes, they show more permanency than long and heavy
RNA. On the other hand some of them that exist out of the vesicles' cover, flow through the blood accompanied by argonaute or surrounded proteins in HDL (High Density Lipoprotein). ${ }^{32}$ Furthermore, many researchers have demonstrated that the expression profiles of miRNAs in various cancers show high tissue specificity. ${ }^{30,33}$ Relying on these characteristics; miRNAs have many requisite features of good biomarkers to be used in diagnosis and follow-up of NASH occurrence and progression.

Since 2006 when the first documents were published about the role of miRNAs in regulation of lipid metabolism in liver tissue, more than 200 papers have been published in this field. ${ }^{34}$ These miRNAs involve in several aspects of lipid and cholesterol metabolism and also some cellular mechanisms such as cell apoptosis.

In this paper we try to list more important miRNAs and pathways involved in physiopathology of NAFLD and NASH.

\section{MiR-122 overexpression is involved in NAFLD:}

MiR-122 is a highly expressed liver-specific miRNA, which encompasses $70 \%$ of liver miRNAs. ${ }^{35,36}$ This miRNA has a critical role in regulation of lipid and cholesterol biosynthesis in hepatocytes. Knockdown of this miRNA leads to reduction of cholesterol and triglyceride levels in plasma up to $26-28 \%$. The results of microarray analysis of liver gene expression in MiR-122 knockout mice have shown changes in expression levels of many genes involved in regulation of lipid and carbohydrate metabolism such as FASN, ACLY, PMVK, SCD1, and ACC2, of them phosphomevalonate kinase (PMVK) function is more characterized in the pathophysiology of fatty liver. ${ }^{37}$ PMVK encodes a peroxisomal enzyme that catalyzes the phosphorylation of mevalonate. ${ }^{38}$ Furthermore miRNA-122 regulate several genes including HMGCS1, HMGCR, and DHCR7, which are involved in homeostasis between fatty acids and cholesterol biosynthesis pathways. ${ }^{39}$ This mir plays an important role in the pathophysiology of NAFLD by regulating several genes critical in cholesterol and lipid hemostasis.

Researchers demonstrated that the impact of MiR-122 not only on lipid metabolism, but also deletion of it promote microsteatosis and inflammation, which lead to poor prognosis of liver cancer. Data demonstrated, delivering of MiR-122 in relevant animal model reversed 
liver inflammation by suppressing MiR-122 targets: chemokine $\mathrm{Ccl} 2$, that recruit $\mathrm{CD} 11 \mathrm{~b}$ hiGr1+inflammatory cells intrahepatically. ${ }^{40}$ This important microrna can reduce progression to cirrhosis and HCC. ${ }^{41}$ Researchers suggested that serum levels of MiR-122 might be an informative biomarker to assess early NAFLD superior to clinical markers classically used to monitor hepatic disease. ${ }^{42}$

MIR-34a induces hepatocyte apoptosis via miR34a/SIRT1/P53 proapoptotic pathway:

MiR-34a is the second most important regulatory miRNA in the liver physiology. This miRNA is located on $1 \mathrm{p} 36^{43}$ and its ectopic expression leads to $\mathrm{G} 1$ arrest and apoptosis. ${ }^{44-46}$

MiR-34a plays an important role in the pathology of fatty liver disease via targeting SIRT1 gene. ${ }^{47}$ SIRT1 stands for sirtuin (silent mating type information regulation 2 homolog) 1, located on 10q21.3, NAD-dependent class III histone deacetylase enzyme, which affects various proteins involved in several cellular pathways ${ }^{48}$ such as deacetylation and thereby deactivation of $\mathrm{p} 53$ protein and regulates several physiological processes such as apoptosis, fat metabolism, and glucose homeostasis. For example in the liver, SIRT1 deacetylates the liver X receptor (it is a nuclear receptor that its role is cholesterol sensor and regulates lipid homeostasis) and suppresses the protein-tyrosine phosphatase 1B (PTP1B), leading to an increase cholesterol transport and decreased insulin resistance, respectively. Researchers theorized that deacetylation of LXRs by SIRT1 might affect atherosclerosis and aging-associated disorders. ${ }^{49}$

Several studies indicate that expression of SIRT1 significantly decreases in NAFLD induced by high-fat diet in rat. ${ }^{50}$

MiR-34a involve in fatty liver disease progression through cell apoptosis induction as a p53 pathway mediator. MiR-34a represses the SIRT1 expression thereby increasing p53 acetylation, which leads to the induction of proapoptotic genes and finally cell death. This is called MiR-34a /SIRT1/P53 proapoptotic pathway. ${ }^{38}$ Studies have revealed that the lack of MiR-34 expression results in resistance of cells against apoptosis induced by P53. ${ }^{51}$

On the other hand, MiR-34a causes apoptosis, cellcycle arrest, and cell senescence by suppressing the expression of antiapoptotic genes such as bcl2, c-MYC, cyclin D, MET, and E2F. As a result, MiR-34 may powerfully responsible for a permanent destruction of cells during suffering from NAFLD. ${ }^{43}$

In conclusion, MiR-34a via targeting SIRT1 gene increases cell death and causes liver tissue injury and researchers show that silencing of MiR-34a in patients with NAFLD could be effective.

\section{MIR-10b regulates lipid storage via targeting of} PPAR-alpha:

Another important miRNA that is effective in the pathogenesis of NAFLD is MIR-10b. It is a non- coding RNA. Its gene is located on chromosome 2 and involved in genes regulation. The direct target of MIR-10b is peroxisome proliferator-activated receptor-alpha (PPARalpha) gene also known as NR1C1 (nuclear receptor subfamily 1 , group $\mathrm{C}$, member 1 ), a nuclear receptor protein that contributes to ketogenesis and a major regulator of lipid metabolism in the liver, located on $22 \mathrm{q} 13 .{ }^{31}$ and alter the expression of a large number of target genes especially the genes involved in various aspects of lipid metabolism. Increased expression of MIR-10b via its effect on PPAR-alpha, causes increased lipid and triglyceride storage in hepatocytes, ${ }^{52}$ which is eventually leads to tissue damage and steatosis of liver.

MIR-33a and MIR-33b coordinate with their host genes to regulate cholesterol biosynthesis:

MIR-33a and MIR-33b regulate genes involved in lipid metabolism too. MIR-33a is located on intron 16 of the SREBP-2 gene and MIR-33b is present in intron 17 of the SREBP-1 gene. ${ }^{53}$ These genes encode transcriptional regulatory factor that contributes to cholesterol uptake and synthesis. ${ }^{38}$ These two miRNAs cooperate with their host genes to regulate cholesterol hemostasis. ${ }^{54}$ These miRNAs regulate genes involved in fatty acid metabolism and insulin signaling such as CROT, CPT1A, SIRT6, HADHA, and PRKAA1. ${ }^{55}$

furthermore up-regulation of these miRNAs and their host genes leads to modulation of HDL, triglycerides, and also insulin signaling, which are the risk factors of metabolic syndrome. ${ }^{56}$ To confirm this role, researchers express that inhibition of these miRNAs in non-human primates cause the increase of HDL and decrease of VLDL levels. ${ }^{57}$ Also the inhibition of these miRNAs 
excitingly cause improving of liver tissue regeneration. ${ }^{58}$

As a result of inquiries, increased cholesterol intake accelerated liver fibrosis in mouse model and the major cause of the accelerated liver fibrosis is free cholesterol (FC) accumulation in hepatic stellate cells (HSCs), which is regulated by sterol regulatory element-binding protein 2 (SREBP2) gene. Data showed that the mRNA expression levels of SREBP2 were significantly higher in HSCs and similarly, the expression levels of LDLR and MIR-33a were significantly higher in the high fat diet-fed mouse groups than in control diet-fed groups leading to increase intake of cholesterol. The results showed that SREBF2 is a bi- functional locus encoding SREBP2 and MIR-33a. Free cholesterol accumulation in HSCs was enhanced mainly by two mechanisms: enhancement of SREBP2 and MIR-33a signaling through the suppression of PPARc signaling. ${ }^{59}$

In conclusion, MIR-33 cluster can be used as a biomarker of liver tissue fibrosis and its inhibition can be considered as of the new therapeutic target for steatosis treatment. ${ }^{53}$

\section{MiRNAs as biomarkers of NAFLD/NASH:}

MiRNAs transfer in body circulation with lipid vesicle like bodies, exosomes, or in combination with HDL. This protects miRNAs from degradation and gives a high stability of these molecules in body fluids. Furthermore this protection system causes a high concordance between tissue and plasma levels of these molecules. Having this characteristics, miRNAs can be used as very powerful biomarkers to diagnosis and follow-up of any disorder, such as fatty liver, with a high specificity and sensitivity. ${ }^{25,28,29}$ The circulating miRNA signature of NAFLD has been explored in several case control studies up to now. ${ }^{60}$ One of these considerable studies explored the potential role of the circulating extracellular vesicles (EVs) as non-invasive biomarker in diet-induced NAFLD mice. They extracted circulating extracellular vesicles and found that the level of EVs correlated with pathological features of hepatic cells especially those enrich with mir-122. ${ }^{61}$ Researchers propose that mir-122 is an early disease biomarker of liver injury and because of its earlier increased level compared with serum ALT, suggested it as an extrahepatic fingerprint of NASH. In NAFLD, they suggested that circulating miRNAs (exactly mir-122) could mirror the histological and molecular process occurring in the liver. ${ }^{62}$

\section{CONCLUSION}

Number of documents about the role of miRNAs in physiopathology of fatty liver disease has increased in recent decade. These small non-coding RNA molecules can regulate a large number of genes and have a huge impact on the initiation and progression of many complex diseases such as fatty liver. MiRNAs can be used as either interesting therapeutic targets to treat fatty liver disease or biomarker to diagnose it. Including pro-anthocyanidin supplementation that decreases postprandial lipemia and MIR-33a or MIR-122 levels can be used in prevention also treatment of NAFLD ${ }^{63}$ In all, further and more experimental and clinical studies are still needed to evaluate miRNAs in diagnosis, follow-up, and even treatment of fatty liver disease.

\section{ETHICAL APPROVAL}

There is nothing to be declared.

\section{CONFLICT OF INTEREST}

The authors declare no conflict of interest related to this work.

\section{REFERENCES}

1. Angulo P. Nonalcoholic fatty liver disease. $N$ Engl J Med 2002;346:1221-31. doi:10.1056/NEJMra011775.

2. Vuppalanchi R, Chalasani N. Nonalcoholic fatty liver disease and nonalcoholic steatohepatitis: Selected practical issues in their evaluation and management. Hepatology 2009;49:306-17. doi:10.1002/hep.22603.

3. Kawamura Y, Arase Y, Ikeda K, Seko Y, Imai N, Hosaka $\mathrm{T}$, et al. Large-scale long-term follow-up study of Japanese patients with non-alcoholic fatty liver disease for the onset of hepatocellular carcinoma. Am J Gastroenterol 2012;107:253-61. doi:10.1038/ajg.2011.327.

4. Shifflet A, Wu GY. Non-alcoholic Steatohepatitis: An Overview. J Formos Med Assoc 2009;108:4-12. doi:10.1016/ S0929-6646(09)60026-7.

5. Alavian SM, Ramezani M, Bazzaz A, Azuzabadi Farahani M, Behnava B, KeshvariM. Frequency of Fatty Liver and Some of Its Risk Factors in Asymptomatic Carriers of HBV Attending the Tehran Blood Transfusion Organization Hepatitis Clinic. Iran J Endocrinol Metabol 2008;10:99-106

6. Sass DA, Chang P, Chopra KB. Nonalcoholic Fatty Liver Disease: A Clinical Review. Dig Dis Sci 2005;50:171-80. doi:10.1007/s10620-005-1267-z.

7. Takaki A, Kawai D, Yamamoto K. Molecular Mecha- 
nisms and New Treatment Strategies for Non-Alcoholic Steatohepatitis (NASH). Int J Mol Sci 2014;15:7352-79. doi:10.3390/ijms 15057352 .

8. Williams CD, Stengel J, Asike MI, Torres DM, Shaw J, Contreras M, et al. Prevalence of Nonalcoholic Fatty Liver Disease and Nonalcoholic Steatohepatitis Among a Largely Middle-Aged Population Utilizing Ultrasound and Liver Biopsy: A Prospective Study. Gastroenterology 2011;140:124-31. doi:10.1053/j.gastro.2010.09.038.

9. Leite NC, Villela-Nogueira CA, Pannain VL, Bottino AC, Rezende GF, Cardoso CR, et al. Histopathological stages of nonalcoholic fatty liver disease in type 2 diabetes: prevalences and correlated factors. Liver Int 2011;31:7006. doi:10.1111/j.1478-3231.2011.02482.x.

10. Kelso LA. Cirrhosis: caring for patients with End-stage liver failure. Nurse Pract 2008;33:24-30. doi:10.1097/01. NPR.0000325976.85753.dd.

11. Das k, Kar P. Non-Alcoholic Steatohepatitis. J Assoc Physicians India 2005;53:195-9.

12. Hoekstra M, van der Sluis RJ, Kuiper J, Van Berkel TJ. Nonalcoholic fatty liver disease is associated with an altered hepatocyte microRNA profile in LDL receptor knockout mice. J Nutr Biochem 2012;23:622-8. doi:10.1016/j.jnutbio.2011.03.005.

13. Dasarathy S, Dasarathy J, Khiyami A, Yerian L, Hawkins C, Sargent R, et al. Double-blind Randomized Placebocontrolled Clinical Trial of Omega 3 Fatty Acids for the Treatment of Diabetic Patients With Nonalcoholic Steatohepatitis. J Clin Gastroenterol 2015;49:137-44. doi: 10.1097/MCG.0000000000000099.

14. Tessitore A, Cicciarelli G, Del Vecchio F, Gaggiano A, Verzella D, Fischietti M, et al. MicroRNA expression analysis in high fat diet-induced NAFLD-NASH-HCC progression: study on C57BL/6J mice. BMC Cancer 2016;16:3. doi:10.1186/s12885-015-2007-1.

15. Tessitore A, Mastroiaco V, Vetuschi A, Sferra R, Pompili S, Cicciarelli G, et al. Development of hepatocellular cancer induced by long term low fat-high carbohydrate diet in a NAFLD/NASH mouse model. Oncotarget 2017;8:5348294. doi:10.18632/oncotarget.18585.

16. Ruby JG, Jan CH, Bartel DP. ntronic MicroRNA precursors that bypass Drosha processing. Nature 2007;448:836. doi:10.1038/nature05983.

17. Krek A, Grün D, Poy MN, Wolf R, Rosenberg L, Epstein EJ, et al. Combinatorial MicroRNA target predictions. Nat Genet 2005;37:495-500. doi:10.1038/ng1536.

18. Khorramshahi V, Mojarad M, Jaripour ME, Darabi H, Azarpazhooh MR, Samadi M, et al. Association of MIR34A Gene Polymorphism rs369892834 with Ischemic and Hemorrhagic stroke risk. Adv Biores 2015;6:71-7.

19. Pasquinelli AE, Reinhart BJ, Slack F, Martindale MQ, Kuroda MI, Maller B, et al. Conservation of the sequence and temporal expression of let-7 heterochronic regulatory RNA. Nature 2000;408:86-9. doi:10.1038/35040556.
20. Gurtan AM, Sharp PA. The Role of miRNAs in Regulating Gene Expression Networks. J Mol Biol 2013;425:3582600. doi:10.1016/j.jmb.2013.03.007.

21. Ambros V. The functions of animal microRNAs. Nature 2004;431:350-5. doi:10.1038/nature02871.

22. Kloosterman WP, Plasterk RH. The diverse functions of microRNAs in animal development and disease. Dev Cell 2006;11:441-50. doi:10.1016/j.devcel.2006.09.009.

23. Alvarez-Garcia I, Miska EA. MicroRNA functions in animal development and human disease. Development 2005;132:4653-62. doi:10.1242/dev.02073.

24. Eslahi A, Daliri ghouchan atigh M, Tabatabaee A, Hosseini NS, Mojarrad M. MicroRNA-124 Overexpression in Associated with Lymph Node Metastasis in Breast Cancer. Cancer Press 2016;2:51-4. doi:10.15562/tcp.16.

25. Schwarzenbach H, Hoon DS, Pantel K. Cell-free nucleic acids as biomarkers in cancer patients. Nat Rev Cancer 2011;11:426-37. doi:10.1038/nrc3066.

26. Mitchell PS, Parkin RK, Kroh EM, Fritz BR, Wyman SK, Pogosova-Agadjanyan EL, et al. Circulating microRNAs as stable blood-based markers for cancer detection. Proc Natl Acad Sci USA 2008;105:10513-8. doi:10.1073/ pnas.0804549105.

27. Mozaheb Z, Hasanzadeh Nazarabadi M, Heydari S, Mojarad M. Cytogenetic alteration and expression of Let-7a microRNA in relation to its target gene NRAS in a family with myelodysplastic syndrome (MDS). Am Soci Clin Oncol 2016.

28. Chen X, Ba Y, Ma L, Cai X, Yin Y, Wang K, et al. Characterization of microRNAs in serum: a novel class of biomarkers for diagnosis of cancer and other diseases. Cell Res 2008;18:997-1006. doi:10.1038/cr.2008.282.

29. Cermelli S, Ruggieri A, Marrero JA, Ioannou GN, Beretta L. Circulating MicroRNAs in Patients with Chronic Hepatitis $\mathrm{C}$ and Non-Alcoholic Fatty Liver Disease. PLoS One 2011;6:e23937. doi:10.1371/journal.pone.0023937.

30. De Guire V, Robitaille R, Tétreault N, Guérin R, Ménard C, Bambace N, et al. Circulating miRNAs as sensitive and specific biomarkers for the diagnosis and monitoring of human diseases: Promises and challenges. Clin Biochem 2013;46:846-60. doi:10.1016/j.clinbiochem.2013.03.015.

31. Murakami Y, Toyoda H, Tanahashi T, Tanaka J, Kumada $\mathrm{T}$, Yoshioka Y, et al. Comprehensive miRNA Expression Analysis in Peripheral Blood Can Diagnose Liver Disease. PLoS One 2012;7:e48366. doi:10.1371/journal. pone. 0048366 .

32. Shen J, Stass SA, Jiang F. MicroRNAs as potential biomarkers in human solid tumors. Cancer Lett 2013;329:12536. doi:10.1016/j.canlet.2012.11.001.

33. Liu R, Chen X, Du Y, Yao W, Shen L, Wang C, et al. Serum MicroRNA Expression Profile as a Biomarker in the Diagnosis and Prognosis of Pancreatic Cancer. Clin Chem 
2012;58:610-8. doi:10.1373/clinchem.2011.172767.

34. Esau C, Davis S, Murray SF, Yu XX, Pandey SK, Pear $\mathrm{M}$, et al. miR-122 regulation of lipid metabolism revealed by in vivo antisense targeting. Cell Metab 2006;3:87-98. doi:10.1016/j.cmet.2006.01.005

35. Li ZY, Xi Y, Zhu WN, Zeng C, Zhang ZQ, Guo ZC, et al. Positive regulation of hepatic miR-122 expression by HNF4alpha. J Hepatol 2011;55:602-11. doi:10.1016/j. jhep.2010.12.023.

36. Lagos-Quintana M, Rauhut R, Yalcin A, Meyer J, Lendeckel W, Tuschl T. Identification of tissue-specific microRNAs from mouse. Curr Biol 2002;12:735-9. doi:10.1016/S09609822(02)00809-6.

37. Esau C, Davis S, Murray SF, Yu XX, Pandey SK, Pear $\mathrm{M}$, et al. miR-122 regulation of lipid metabolism revealed by in vivo antisense targeting. Cell Metab 2006;3:87-98. doi:10.1016/j.cmet.2006.01.005.

38. Vincent R, Sanyal A. Recent Advances in Understanding of NASH: MicroRNAs as Both Biochemical Markers and Players. Curr Pathobiol Rep 2014;2:109-15. doi:10.1007/ s40139-014-0049-8.

39. Krützfeldt J, Rajewsky N, Braich R, Rajeev KG, Tuschl $\mathrm{T}$, Manoharan $\mathrm{M}$, et al. Silencing of microRNAs in vivo with "antagomirs". Nature 2005;438:685-9. doi:10.1038/ nature 04303 .

40. Hsu Sh, Wang B, Kota J, Yu J, Costinean S, Kutay H, et al. Essential metabolic, anti-inflammatory, and antitumorigenic functions of miR-122 in liver. J Clin Invest 2012;122:2871-83. doi:10.1172/JCI63539.

41. Bandiera S, Pfeffer S, Baumert TF, Zeisel MB. miR-122-a key factor and therapeutic target in liver disease. $J$ Hepatol 2015;62:448-57. doi:10.1016/j.jhep.2014.10.004.

42. Yamada H, Ohashi K, Suzuki K, Munetsuna E, Ando Y, Yamazaki M, et al. Longitudinal study of circulating miR-122 in a rat model of non-alcoholic fatty liver disease. Clin Chim Acta 2015;446:267-71. doi:10.1016/j. cca.2015.05.002.

43. Hermeking H. The miR-34 family in cancer and apoptosis. Cell Death Differ 2010;17:193-9. doi:10.1038/cdd.2009.56.

44. He L, He X, Lim LP, de Stanchina E, Xuan Z, Liang Y, et al. A microRNA component of the p53 tumour suppressor network. Nature 2007;447:1130-4. doi:10.1038/ nature 05939.

45. Tarasov V, Jung P, Verdoodt B, Lodygin D, Epanchintsev A, Menssen A, et al. Differential regulation of microRNAs by $\mathrm{p} 53$ revealed by massively parallel sequencing: miR-34a is a p53 target that induces apoptosis and G1-arrest. Cell Cycle 2007;6:1586-93. doi:10.4161/cc.6.13.4436.

46. Bommer GT, Gerin I, Feng Y, Kaczorowski AJ, Kuick R, Love RE, et al. p53-mediated activation of miRNA34 candidate tumor-suppressor genes. Curr Biol 2007;17:1298307. doi:10.1016/j.cub.2007.06.068.
47. Szabo G, Bala S. MicroRNAs in liver disease. Nat Rev Gastroenterol Hepatol 2013;10:542-52. doi:10.1038/ nrgastro.2013.87.

48. Brooks $\mathrm{CL}, \mathrm{Gu}$ W. How does SIRT1 affect metabolism, senescence and cancer? Nat Rev Cancer 2009;9:123-8. doi:10.1038/nrc2562.

49. Li X, Zhang S, Blander G, Tse JG, Krieger M, Guarente L. SIRT1 deacetylates and positively regulates the nuclear receptor LXR. Mol Cell 2007;28:91-106. doi: 10.1016/j. molcel.2007.07.032.

50. Deng XQ, Chen LL, Li NX. The expression of SIRT1 in nonalcoholic fatty liver disease induced by high-fatdiet in rats. Liver Int 2007;27:708-15. doi:10.1111/j.14783231.2007.01497.x.

51. Ji Q, Hao X, Meng Y, Zhang M, DeSano J, Fan D, et al. Restoration of tumor suppressor miR-34 inhibits human p53-mutant gastric cancer tumorspheres. BMC Cancer 2008;8:266. doi:10.1186/1471-2407-8-266.

52. Zheng L, Lv Gc, Sheng J, Yang Yd. Effect of miRNA-10b in regulating cellular steatosis level by targeting PPARalpha expression, a novel mechanism for the pathogenesis of NAFLD. J Gastroenterol Hepatol 2010;25:156-63. doi:10.1111/j.1440-1746.2009.05949.x.

53. Dávalosa A, Goedekea L, Smibertb P, Ramíreza CM, Warriera NP, Andreoa U, et al. miR-33a/b contribute to the regulation of fatty acid metabolism and insulin signaling. Proc Natl Acad Sci USA 2011;108:9232-7. doi:10.1073/ pnas. 1102281108 .

54. Horton JD, Goldstein JL, Brown MS. SREBPs: activators of the complete program of cholesterol and fatty acid synthesis in the liver. J Clin Invest 2002;109:1125-31. doi: 10.1172/JCI15593.

55. Kim HS, Xiao C, Wang RH, Lahusen T, Xu X, Vassilopoulos A, et al. Hepatic-specific disruption of SIRT6 in mice results in fatty liver formation due to enhanced glycolysis and triglyc-eride synthesis. Cell Metab 2010;12:224-36. doi:10.1016/j.cmet.2010.06.009.

56. Rayner KJ, Suárez Y, Dávalos A, Parathath S, Fitzgerald ML, Tamehiro N, et al. MiR-33 contributes to the regulation of cholesterol homeostasis. Science 2010;328:1570-3. doi:10.1126/science. 1189862 .

57. Rayner KJ, Esau CC, Hussain FN, McDaniel AL, Marshall $\mathrm{S}$, van Gils JM, et al. Inhibition of miR-33a/b in non-human primates raises plasma HDL and lowers VLDL triglycerides. Nature 2011;478:404-7. doi:10.1038/nature10486.

58. Tryndyak VP, Ross SA, Beland FA, Pogribn IP. DownRegulation of the microRNAs miR-34a, miR-127, and miR-200b in Rat Liver During Hepatocarcinogenesis Induced by a Methyl-Deficient Diet. Mol Carcinog 2009;48:479-87. doi:10.1002/mc.20484.

59. Tomita K, Teratani T, Suzuki T, Shimizu M, Sato H, Narimatsu K, et al. Free cholesterol accumulation in hepatic stellate cells: mechanism of liver fibrosis aggrava- 
tion in nonalcoholic steatohepatitis in mice. Hepatology 2014;59:154-69. doi:10.1002/hep.26604.

60. Pirola CJ, Sookoian S. Multiomics biomarkers for the prediction of nonalcoholic fatty liver disease severity. World J Gastroenterol 2018;24:1601-15. doi:10.3748/wjg.v24. i15.1601.

61. Povero D, Eguchi A, Li H, Johnson CD, Papouchado $\mathrm{BG}$, Wree A, et al. Circulating extracellular vesicles with specific proteome and liver microRNAs are potential biomarkers for liver injury in experimental fatty liver disease. PloS One 2014;9:e113651. doi:10.1371/journal. pone.0113651.

62. Pirola CJ, Fernández Gianotti T, Castaño GO, Mallardi P, San Martino J, Mora Gonzalez Lopez Ledesma M, et al. Circulating microRNA signature in non-alcoholic fatty liver disease: from serum non-coding RNAs to liver histology and disease pathogenesis. Gut 2015;64:800-12. doi:10.1136/gutjnl-2014-306996.

63. Baselga-Escudero L, Blade C, Ribas-Latre A, Casanova E, Salvadó MJ, Arola L, et al. Chronic supplementation of proanthocyanidins reduces postprandial lipemia and liver miR-33a and miR-122 levels in a dose-dependent manner in healthy rats. $J$ Nutr Biochem 2014;25:151-6. doi:10.1016/j.jnutbio.2013.09.014. 